\title{
Crystal nucleation and metastable bcc phase in charged colloids: A molecular dynamics study
}

Xinqiang Ji, Zhiwei Sun, Wenze Ouyang, and Shenghua Xu

Citation: The Journal of Chemical Physics 148, 174904 (2018); doi: 10.1063/1.5016235

View online: https://doi.org/10.1063/1.5016235

View Table of Contents: http://aip.scitation.org/toc/jcp/148/17

Published by the American Institute of Physics

\section{Articles you may be interested in}

Bulk viscosity of molecular fluids

The Journal of Chemical Physics 148, 174504 (2018); 10.1063/1.5022752

Time correlation functions of simple liquids: A new insight on the underlying dynamical processes

The Journal of Chemical Physics 148, 174501 (2018); 10.1063/1.5025120

High-density equation of state for a two-dimensional Lennard-Jones solid

The Journal of Chemical Physics 148, 174505 (2018); 10.1063/1.5029488

Theoretical prediction of crystallization kinetics of a supercooled Lennard-Jones fluid

The Journal of Chemical Physics 148, 204506 (2018); 10.1063/1.5021944

Communication: Glass transition and melting lines of an ionic liquid

The Journal of Chemical Physics 148, 171101 (2018); 10.1063/1.5030083

Gas solubility in dilute solutions: A novel molecular thermodynamic perspective

The Journal of Chemical Physics 148, 174502 (2018); 10.1063/1.5023893

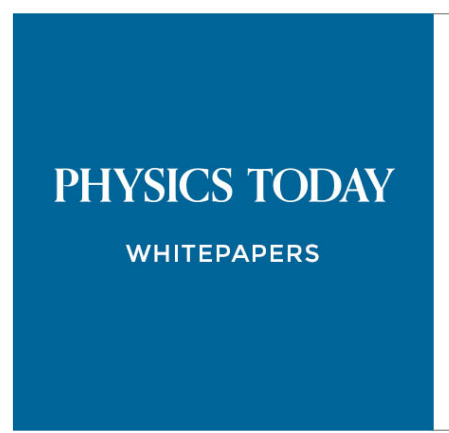




\title{
Crystal nucleation and metastable bcc phase in charged colloids: A molecular dynamics study
}

\author{
Xinqiang Ji, ${ }^{1,2}$ Zhiwei Sun, ${ }^{1}$ Wenze Ouyang, ${ }^{1, a)}$ and Shenghua $\mathrm{Xu}^{1,2}$ \\ ${ }^{1}$ Key Laboratory of Microgravity (National Microgravity Laboratory), Institute of Mechanics, \\ Chinese Academy of Sciences, Beijing 100190, China \\ ${ }^{2}$ School of Engineering Science, University of Chinese Academy of Sciences, Beijing 100049, China
}

(Received 17 November 2017; accepted 15 April 2018; published online 3 May 2018)

\begin{abstract}
The dynamic process of homogenous nucleation in charged colloids is investigated by brute-force molecular dynamics simulation. To check if the liquid-solid transition will pass through metastable bcc, simulations are performed at the state points that definitely lie in the phase region of thermodynamically stable fcc. The simulation results confirm that, in all of these cases, the preordered precursors, acting as the seeds of nucleation, always have predominant bcc symmetry consistent with Ostwald's step rule and the Alexander-McTague mechanism. However, the polymorph selection is not straightforward because the crystal structures formed are not often determined by the symmetry of intermediate precursors but have different characters under different state points. The region of the state point where bcc crystal structures of large enough size are formed during crystallization is narrow, which gives a reasonable explanation as to why the metastable bcc phase in charged colloidal suspensions is rarely detected in macroscopic experiments. Published by AIP Publishing. https://doi.org/10.1063/1.5016235
\end{abstract}

\section{INTRODUCTION}

Crystallization is an important phenomenon which exists in many fields such as biology, chemistry, and materials science. ${ }^{1-4}$ At the onset of crystallization, nucleation is a fundamental process for the crystal structures that determine the functions. ${ }^{5}$ Therefore a deep understanding of nucleation will be helpful to have a control over the quality of the crystal. For instance, the undesired crystal formation can be prevented while the desired one can be promoted with the knowledge of the nucleation mechanism.

To explain the mechanism of crystal nucleation, many different criteria have been proposed. The classical nucleation theory is the most widely used one because of its simplicity and success in explaining many experimental results. According to this theory, the nucleation may possibly happen anywhere in the liquid and the nucleus will not keep growing to the final state until it reaches a critical size, which means that a free-energy barrier should be overcome. However, many researches have shown that there are intermediate states during the crystallization indicating that the crystal nucleation is not just a one-step process. ${ }^{4,6-26}$ In 1897 , Ostwald proposed a credited criterion named Ostwald's step rule stating that the first formed crystal structure should be the one whose free energy is closest to that of the liquid. ${ }^{27}$ Alexander and McTague afterwards demonstrated that the body-centered cubic (bcc) phase, at least for weakly firstorder phase transition in three dimensional simple system, is favored. ${ }^{28}$ Although a variety of different nucleation scenarios are consistent with Ostwald's step rule, ${ }^{4,21-26}$ there

a)Email: oywz@imech.ac.cn are still some exceptions indicating that Ostwald's step rule is not yet universally accepted..$^{15}$ Another intermediate state, which is the relatively ordered structure and so-called precursor, has recently been found to be produced before the nucleation. Among those previous studies discussing the roles of precursors on crystal nucleation, there are basically three types of preordering: ${ }^{29}$ crystallization involving composition fluctuations, ${ }^{4-9}$ the role of density fluctuations in crystal nucleation, ${ }^{10,11}$ and the roles of orientational ordering in crystal nucleation. ${ }^{12,13,16-18,20,30,31}$ Tanaka and co-workers, based on their observations of crystal nucleation, even suggested further that a typical crystallization process was not a discrete two-step process but a continuous one at the microscopic level. ${ }^{20}$

In order to study the nucleation mechanism, here we consider the charged colloidal suspensions, as they have larger temporal and spatial scales than atoms and molecules making it easier to observe the nucleation process due to much more measurement means available. ${ }^{32-34}$ Our recent experiments of the reflection spectrum showed that there was a volume-fraction window for the observation of the metastable bcc structure. ${ }^{14}$ However, it is impossible to use such a conventional macro measurement to detect precursors in nucleation. In this work, we will perform molecular dynamics (MD) simulations to microscopically study the crystallization of charged colloids as well as to explain the narrow region of the metastable bcc phase observed in our macroscopic experiments.

We use a Yukawa potential to mimic the electrostatic interaction of charged colloidal particles. Its phase diagram (see Fig. 1), where there are both bcc and face-centered-cubic (fcc) phase regions, has already been obtained previously (e.g., Ref. 35). As the Yukawa system is a good option to 


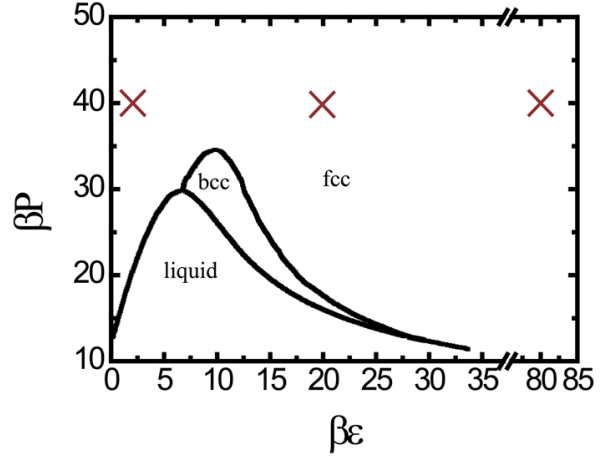

FIG. 1. Phase diagram of hard-core Yukawa potential with the inverse screening length $\kappa=5$, produced from the data in Ref. 35. $\beta=1 / k_{\mathrm{B}} T$. The state points concerned in the present work are marked with crosses: fixed pressure $P=40$ and in the order of increasing the contact energy $\varepsilon=2,20$, and 80 , respectively.

study the liquid-solid phase transition, a variety of numerical simulations actually have been carried out. ${ }^{17,36-39}$ However, little attempt has been made on the dynamics process of its crystal nucleation and the related subtle crystallization mechanism is not yet understood completely.

The aim of this paper is to further discover the mechanism of crystal nucleation in charged colloids. We perform bruteforce molecular dynamic (MD) simulation that is closer to the real crystallization process to investigate the dynamic nucleation in the region of the solid phase of the Yukawa model system. In Sec. II, we introduce the details of our model and simulation. Then in Sec. III, we report the simulation results under different conditions. The final part is the conclusion of this work.

\section{MODEL AND METHODS OF SIMULATION}

\section{A. Colloid model}

Based on the seminal work of Derjaguin, Landau, Verwey, and Overbeek which is known as DLVO theory, ${ }^{40}$ the effective interaction of charged colloidal particles is modeled by a repulsive Yukawa potential

$$
U_{\text {Yukawa }}(r)=\varepsilon \frac{\exp (-\kappa(r / \sigma-1))}{r / \sigma} .
$$

The excluded volume of particles can be represented by a Weeks-Chandler-Anderson (WCA) potential

$$
U_{W C A}(r)=\left\{\begin{array}{ll}
4 \varepsilon_{W}\left[\left(\frac{\sigma}{r}\right)^{12}-\left(\frac{\sigma}{r}\right)^{6}+\frac{1}{4}\right], & r<2^{\frac{1}{6}} \sigma \\
0 & \text { else }
\end{array} .\right.
$$

Here $\sigma$ is the particle diameter, $\varepsilon$ is the intensity of the repulsive Yukawa potential, $\kappa$ denotes the inverse screening length, and $\varepsilon_{\mathrm{W}}$ is the intensity of WCA potential. To combine these two equations, the full terms of the potential are given by $^{37}$

$$
U(r)=U_{\text {Yukawa }}(r)+U_{W C A}(r) .
$$

We define $\sigma$ and $\varepsilon_{\mathrm{W}}$ to be the units of length and energy, respectively. Also, we set the mass of each colloid $M=1$ and $k_{\mathrm{B}} T=1$.

\section{B. Bond-orientational order parameters}

To investigate the dynamics of crystal nucleation, we characterize the structure of each particle using bond-orientational order parameters. For the system of long-range repulsions such as charged colloids, the bond-orientational analysis cannot be done in a straightforward manner because of the difficulty in the proper assignment of nearest-neighbor particles. ${ }^{41,42}$ So we apply the Voronoi diagram to define the nearest neighbors and normalize bond-orientational parameter via the surface area of each Voronoi cell. ${ }^{41,42}$ Here a complex vector of a particle $i$ is defined as

$$
q_{l m}(i)=\frac{1}{N_{n b}(i)} \sum_{j=1}^{N_{n b}(i)} \frac{A(j)}{A} Y_{l m}\left(\mathbf{r}_{i j}\right),
$$

where $N_{n b}(i)$ is the number of the nearest neighbors of particle $i, l$ is a free integer parameter, $m$ is an integer that runs from $m=-l$ to $m=+l, A(j)$ is the surface area of the Voronoi cell facet separating particle $i$ and its neighbor particle $j, A$ is the total surface area $\mathrm{A}=\sum_{j=1}^{N_{n b}(i)} A(j), Y_{l m}\left(\boldsymbol{r}_{i j}\right)$ is the spherical harmonics function, and $\boldsymbol{r}_{i j}$ is the vector from particle $i$ to particle $j$.

For the identification of the solid particles, an approach proposed by Frenkel and co-workers is applied. ${ }^{21,22}$ First we use a normalized complex vector

$$
d_{6 m}(i)=\frac{q_{6 m}(i)}{\left[\sum_{m=-6}^{6}\left|q_{6 m}\right|^{2}\right]^{1 / 2}} .
$$

Then we calculate a scalar

$$
S_{i j}=\sum_{m=-6}^{6} d_{6 m}(i) \cdot d_{6 m}^{*}(j),
$$

where the * indicates complex conjugation. This scalar characterizes the correlation between the particles $i$ and $j$. If $S_{i j}$ exceeds a threshold, typically 0.7 , we consider particle $i$ and $j$ to be connected. If the number of connections of a particle surpasses a given value, typically 7 , this particle is identified as solid. Otherwise, it is a liquid particle.

Further development on the accuracy of crystalline structure identification is made by Lechner and Dellago. ${ }^{43}$ They change the $q_{l m}$ into an averaged form

$$
\bar{q}_{l m}(i)=\frac{1}{N_{n b}(i)} \sum_{j=0}^{N_{n b}(i)} q_{l m}(j) .
$$

It sums up all the local order parameters of all neighbors around particle $i$ and particle $i$ itself. Then, the local order parameters of the averaged version are

$$
Q_{l}(i)=\left(\frac{4 \pi}{2 l+1} \sum_{m=-l}^{l}\left|\bar{q}_{l m}(i)\right|^{2}\right)^{1 / 2}
$$

and

$$
W_{l}(i)=\sum_{m_{1}+m_{2}+m_{3}=0}\left(\begin{array}{ccc}
l & l & l \\
m_{1} & m_{2} & m_{3}
\end{array}\right) \times \frac{\bar{q}_{l m_{1}}(i) \bar{q}_{l m_{2}}(i) \bar{q}_{l m_{3}}(i)}{\left(\sum_{m=-l}^{l}\left|\bar{q}_{l m}(i)\right|^{2}\right)^{3 / 2}},
$$

where the term in parentheses is the Wigner 3-j symbol. The integers $m_{1}, m_{2}$, and $m_{3}$ range from $-l$ to $+l$ with the precondition that the sum of the three integers equals to 0 . 
TABLE I. Parameters used in MD simulations: the contact energy $\varepsilon$, the pressure $P$, the number density of initial configuration $\rho_{A}$ and that of final configuration $\rho_{B}$, the volume fraction of initial configuration $\eta_{A}$ and that of final configuration $\eta_{B}$ where the system is almost crystallized completely, the time step $\delta t$, and the total steps stepLimit.

\begin{tabular}{lccccccc}
\hline \hline$\varepsilon$ & $P$ & $\rho_{A}$ & $\rho_{B}$ & $\eta_{A}$ & $\eta_{B}$ & $\delta t$ & stepLimit \\
\hline 2 & 40 & 1.0912 & 1.1332 & 0.5714 & 0.5933 & 0.005 & $10^{5}$ \\
20 & 40 & 0.6255 & 0.6563 & 0.3275 & 0.3436 & 0.005 & $10^{5}$ \\
80 & 40 & 0.3936 & 0.4374 & 0.2061 & 0.2290 & 0.005 & $10^{5}$ \\
\hline \hline
\end{tabular}

In this work, the identification of crystal structure is as follows. A crystal particle is first identified with the method presented by Frenkel and co-workers. ${ }^{21,22}$ Then it will be considered to be ${ }^{30}$ (i) fcc as $W_{6}<0$ and $W_{4} \leq 0$; (ii) hexagonalclose-packed (hcp) as $W_{6}<0$ and $W_{4}>0$; (iii) bcc as $W_{6} \geq 0$.

\section{Brute-force simulation}

We use the method of brute-force MD simulation to investigate the nucleation evolution over time. ${ }^{44}$ The system consists of 8000 particles in a three dimensional box with periodic boundary conditions. Simulations are carried out in the isothermal-isobaric ensemble realized via a Berendsen thermostat and barostat. ${ }^{45}$ We set the cutoff distance of the Yukawa potential $r_{c}=2.5$. The equation of motion is integrated using the velocity Verlet algorithm. ${ }^{46,47}$ Combining the crystal identification method mentioned above, we will yield the information of the phase behavior during the nucleation process. The other parameters used in MD simulation are given in Table I.

In brute-force simulations, the state points that are too close to the liquid-solid coexistence line can rarely be nucleated. In order to find the phase transition within the typical time scales of MD simulation, we need to start the simulations at the state points of relatively strong supersaturation. Here three typical state points in the phase diagram where the stable phase is fcc are specifically concerned (see Fig. 1). Before the crystallization, the system is relaxed to eliminate the effect of initial configuration. For a better statistics, we conduct 100 independent MD simulations at each state point.

\section{RESULTS AND DISCUSSION}

\section{A. Precursor formation}

As a fundamental process of crystallization, the formation of precursors has been observed in various systems. ${ }^{4,8-13,16,17}$ In the picture of precursor-mediated crystallization, precursors act as the seeds of nucleation. Before the nucleation takes place, some clusters of precursors appear first in the system. Afterwards, a few crystal particles emerge inside the precursors. When the nuclei grow, the precursors are always wetting on the surface of nuclei and waiting to become crystals with a simultaneous supplementation from outer particles. During the crystal nucleation, the nuclei are roughly spherical and the shape of precursors is like an attached shell (see, e.g., the latter snapshots shown in Fig. 7). As previous studies have suggested that there are correlations between the location of critical nucleus and $Q_{6}$ order parameter, ${ }^{13,16,18,20,31}$ we define the liquid particles with the character of $Q_{6} \geq 0.3$ as the precursors. Notice here that the threshold of $Q_{6}$ for precursors is just chosen arbitrarily, and that it can of course be chosen as other values (e.g., 0.28 and 0.29 ) close to 0.3 . A little different value of threshold may influence the number of precursors slightly but the conclusion will not change substantially (see also Fig. 4).

One may ask why the precursors are not identified by the local densities. This is actually related to an open issue of crystal nucleation, i.e., "bond-order-first" or "density-first." Up to now, there has been some debate about whether the nuclei emerge in dense precursors or in bond-orientational-ordered precursors. ${ }^{29}$ Here we have calculated the average local density of precursors $\bar{\rho}_{\text {precursor }}$ with different threshold values of $Q_{6}\left(Q_{6}{ }^{t h r}\right)$, to find a weakly linear coupling between $\bar{\rho}_{\text {precursor }}$ and $Q_{6}{ }^{t h r}$ (see Fig. 2). The observation that $\bar{\rho}_{\text {precursor }}$ does not increase significantly with $Q_{6}{ }^{\text {thr }}$ suggests that the bondorientational order parameter $Q_{6}$ plays a more important role than the density, thus we believe that it is better to choose $Q_{6}$ to identify the precursors.

Similar to the crystal identification, the structural symmetries of precursors can also be characterized on the $W_{6}-W_{4}$ plane. For a precursor particle, it is (i) fcc-like as $W_{6}<0$ and $W_{4} \leq 0$; (ii) hcp-like as $W_{6}<0$ and $W_{4}>0$; (iii) bcc-like as $W_{6} \geq 0$. Then we present the investigation of precursors during MD simulations. For different initial configurations, the nucleation event may possibly occur at different times. Here we make the statistics started from a similar nucleation level where the number of solid particles is about 100. Figure 3, which summarizes the statistic variation of precursors with different symmetries over time, shows that the bcc-like precursors are always dominant. Such an observation of the predominantly bcc-ordered structure in precursors is consistent with Ostwald's step rule ${ }^{27}$ and the Alexander-McTague scenario. $^{28}$

In order for a further investigation, we calculate the fractional composition of three kinds of precursors with different $Q_{6}{ }^{t h r}$ (ranging from 0.1 to 0.32 ), as shown in Fig. 4. At all the state points considered, the bcc ordering is always favored as expected. Specifically the fraction of bcc-like particles is kept almost constant under a fairly range of

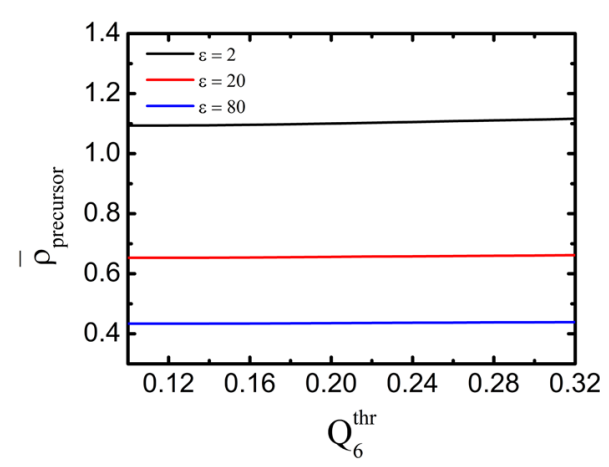

FIG. 2. Average local density of precursors as a function of the threshold value of $Q_{6}$ for $P=40$. The data are taken from the nucleation stage when the total number of solid particles in the system is about 100 . 

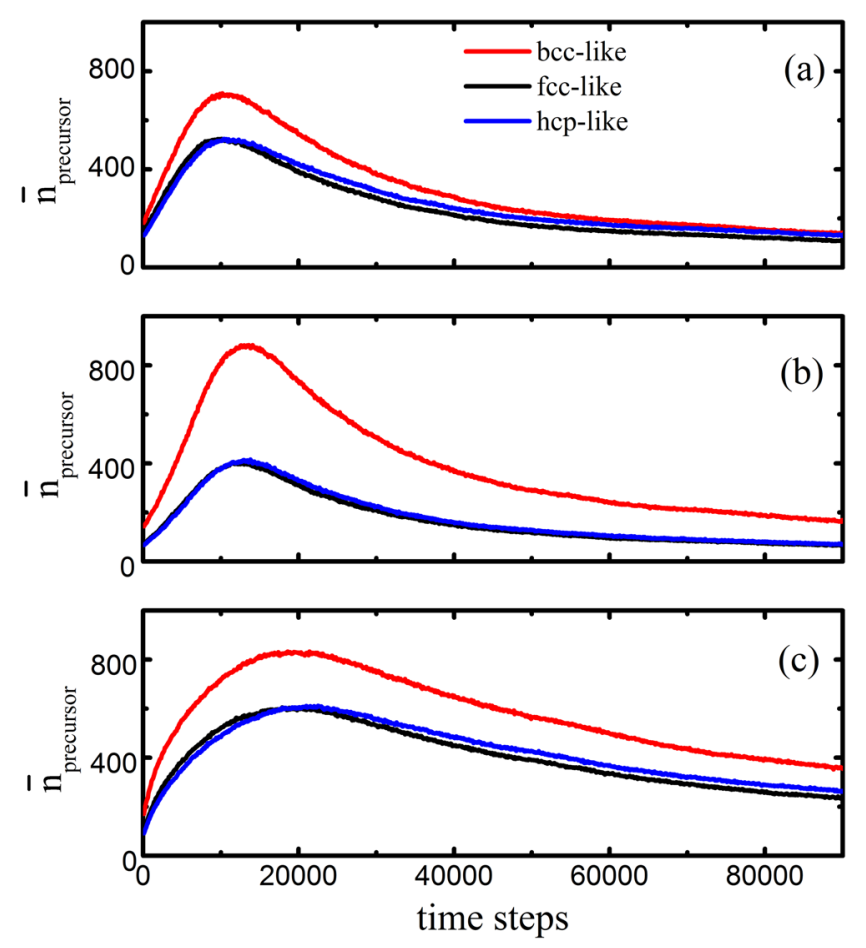

FIG. 3. Average number of precursor particles during crystallization for $P=40$. (a) $\varepsilon=2$; (b) $\varepsilon=20$; (c) $\varepsilon=80$. Red, black, and blue lines represent the curves of bcc-like, fcc-like, and hcp-like precursor particles, respectively.

$Q_{6}{ }^{t h r}$. When increasing $Q_{6}{ }^{t h r}$, the fraction of fcc-like particles increases but the fraction of hcp-like particles decreases slightly while the fraction of bcc-like particles begins to decrease after a big enough value of $Q_{6}{ }^{t h r}$. Many previous studies have shown that the bond-orientational order parameter $Q_{6}$ plays an important role in the crystallization process
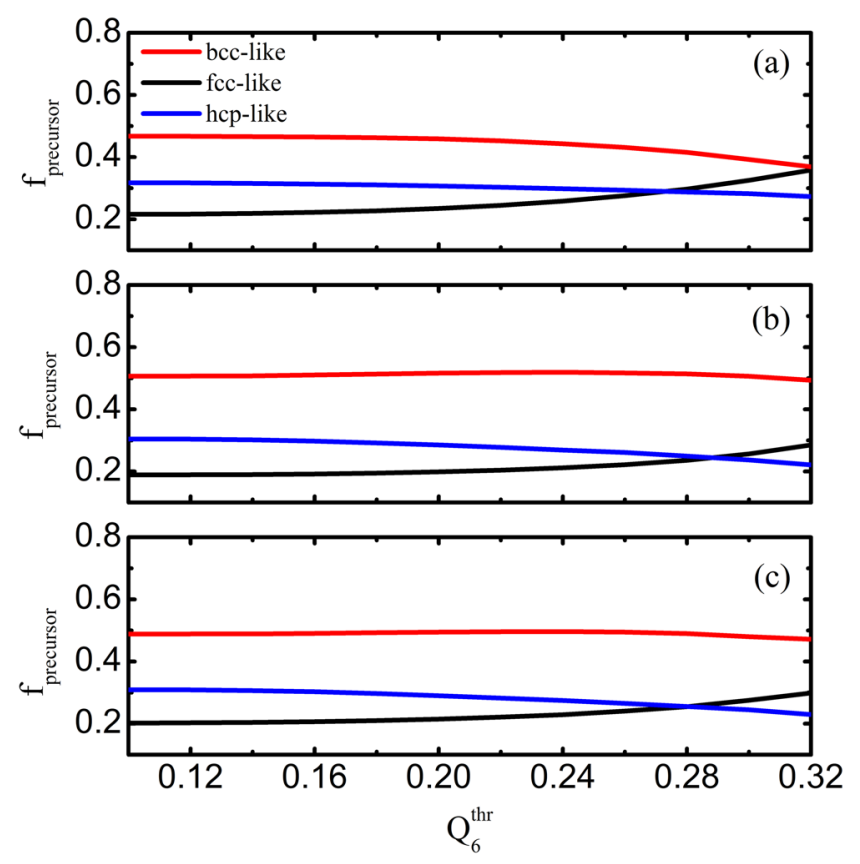

FIG. 4. Fractional composition of three kinds of precursors as a function of the threshold value of $Q_{6}$ for $P=40$. (a) $\varepsilon=2$; (b) $\varepsilon=20$; (c) $\varepsilon=80$. The data are taken from the nucleation stage when the total number of solid particles in the system is about 100. Red, black, and blue lines represent the curves of bcc-like, fcc-like, and hcp-like precursor particles, respectively. as it triggers the crystal nucleation. ${ }^{19,20}$ Therefore the observations shown in Fig. 4 also imply a tendency of the crystal nucleation.

Here we would like to mention that our findings for precursors seem to be different from those of Tan et al. who reported that precursors were mostly hcp-like but not bcc-like. ${ }^{12}$ This discrepancy is probably due to a different approach of the identification of local symmetry. As noticed in Ref. 42, care should be taken for the identification of the neighborhood configuration of charged colloids and the use of the $W_{6}$ parameter may be crucial for local symmetry assignment. Furthermore, the nuclei have a dominant bcc symmetry under the condition of weakly first-order transition (see Secs. III B and III C). With the consistency of the local rotational symmetry between the liquid and crystals, the interfacial energy can be reduced significantly which is favorable for the crystal nucleation. Thus it is physically more reasonable that the locally favored structures in the preordered precursors prefer to have a bcc-type symmetry.

As the crystallization process starts, the particles become more and more ordered with an increase of $Q_{6}$. It is interesting to know the polymorph selection, i.e., what crystal structures would preferentially be formed. From the predominantly bcc-ordered structures of precursors, one may expect that the structures of crystal nucleation, which will in principle be compatible with those of the precursors, should also have more bcc crystals than other kinds of crystals. However, the crystal nucleation pathway, which will be described in Subsection III B, is not that simple as the precursor formation indicating that precursors and crystal nucleation have different mechanisms.

\section{B. Crystal nucleation}

We keep the pressure $P=40$ and vary the contact energy value $\varepsilon$ to ensure that the state point is in the stable fcc phase region and far enough from the liquid-solid line so as to make the crystallization success in a direct MD simulation. By varying $\varepsilon$, we can study the crystal nucleation under the conditions from weak to strong charge of colloidal particles. We have seen that under different state points the polymorph selection of crystal nucleation is rather different, as is suggested by Fig. 5 where the relationship between the nucleus size and the average number of various solid particles is displayed.

For $\varepsilon=2$ and $P=40$, fcc particles are always majority during the nucleation and crystal growth process [see also a typical sample shown in Fig. 6(a)]. This is in agreement with the previous observations in weakly charged colloids where the crystallization showed a strong fcc symmetry. ${ }^{36,37}$ Here there is a subtle detail different from the result of Ref. 36. In Ref. 36, the nucleation of bcc crystal was uniquely favored because the small or pre-critical nuclei had the dominant bcc structure and the core of large nuclei transformed from the bcc to fcc structure. Actually such a subtle difference is due to the fact that the crystal identification we take is stricter. In other words, many of the solid particles in Ref. 36 are presumably the preordered liquids (precursors) in our simulation. 

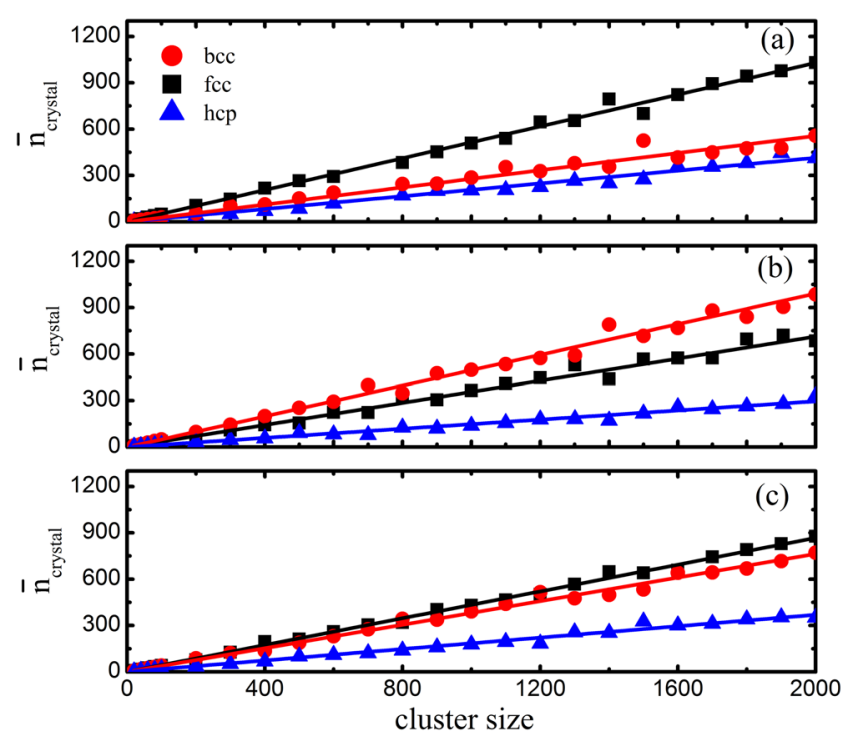

FIG. 5. Average number of different crystal particles in the nuclei as a function of the cluster size for $P=40$. (a) $\varepsilon=2$; (b) $\varepsilon=20$; (c) $\varepsilon=80$. Red, black, and blue symbols represent the curves of bcc, fcc, and hcp particles, respectively. The scatters are the averaged results and the solid lines are a guide to the eye.

Figure 5(b) corresponds to the crystal nucleation for $\varepsilon=20$ and $P=40$. Unlike the former picture of $\varepsilon=2$ and $P=40$, the bcc crystal is always favored despite fcc is the stable phase which complies with Ostwald's step rule and the Alexander-McTague mechanism. As the nucleus grows, the proportions of both fcc and bcc particles do not change much and the bcc crystal always remains dominant. Such a phenomenon, which has also been investigated in Ref. $37(\varepsilon=20$, $P=25.37$ less than ours), is mainly because of the fact that the free-energy barrier between a bulk bcc phase and a bulk fcc phase is too high to make the bcc-fcc transition possible within the time scale of simulation.

According to Refs. 36 and 37, the free-energy barrier of bcc-fcc transition is dependent on the contact energy $\varepsilon$. When $\varepsilon$ is decreased, the free-energy barrier of bcc-fcc transition can become low enough to be overcome spontaneously. Such an explanation can resolve why the bcc particles of $\varepsilon=2$ are less than those of $\varepsilon=20$. But continuing to increase $\varepsilon$, we find that fcc is dominant over bcc again [see Figs. 5(c) and 6(c) for $\varepsilon=80$ and $P=40]$. This indicates that the nucleation pathway is rather more complex than expected. On the other hand, the state points with high enough supersaturation (large $\varepsilon$ and high $P$ ) may run into the spinodal regime where the free energy barrier approaches 0 , making the kinetic pathway through this regime more favorable than others. When the metastable bcc structure is in this regime, its lifetime will be very short and the transition from metastable bcc to stable fcc can be done instantaneously, and in this case, it looks as if the predominant fcc structure is formed directly
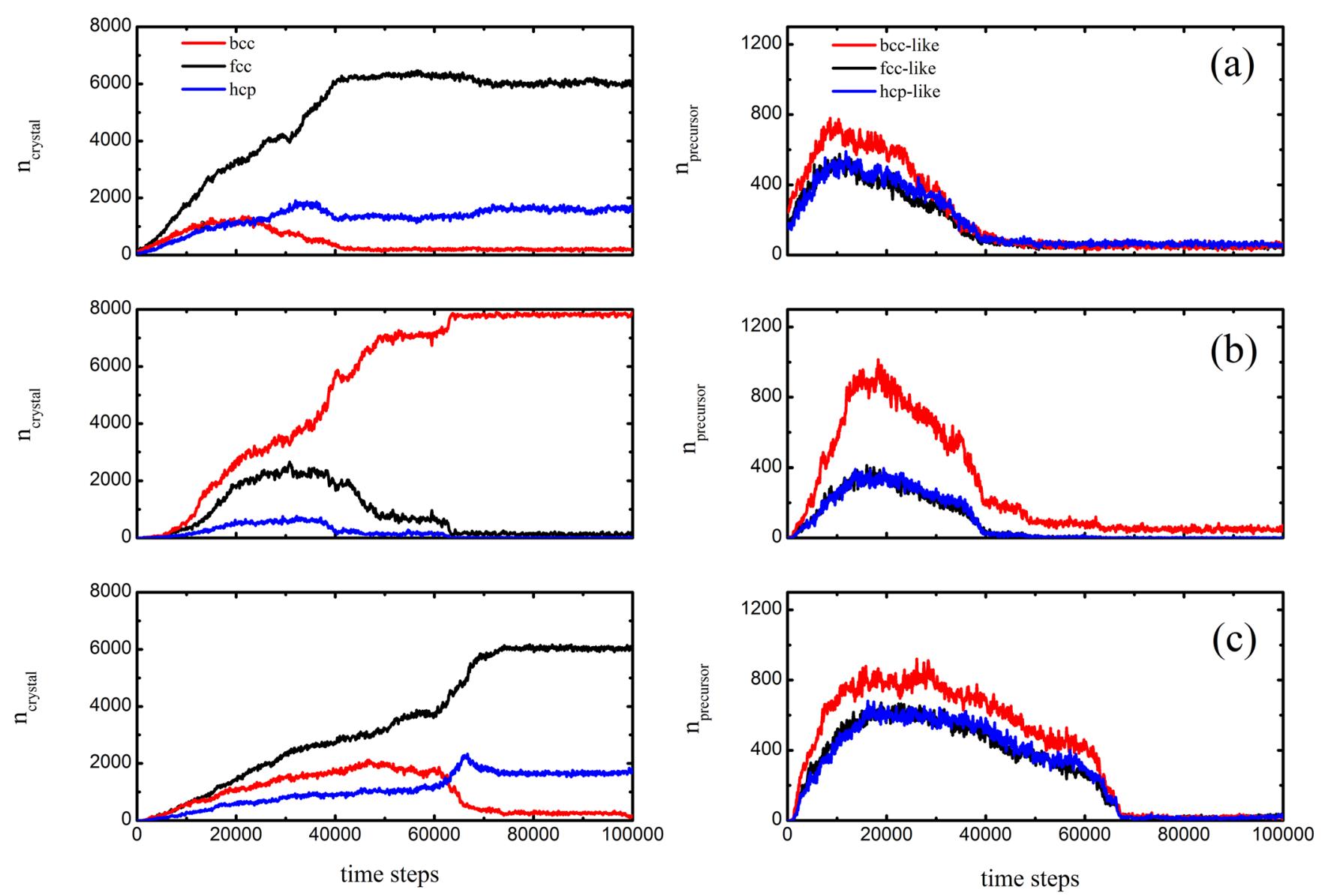

FIG. 6. The number of crystal (left) and precursor (right) particles during a typical MD simulation for $P=40$. (a) $\varepsilon=2$; (b) $\varepsilon=20$; (c) $\varepsilon=80$. Red, black, and blue lines represent the curves of bcc, fcc, and hcp particles, respectively. 
without the intermediate bcc structure emergence. ${ }^{15}$ As the spinodal regime is unknown, the present observation that fcc is dominant over bcc is also possible due to the spinodal decomposition.

Based on the above discussion, we can conclude that the favored phase during nucleation depends on the state point where the system lies. Notice that during the nucleation process, the bcc proportion only dominates at intermediate (e.g., $\varepsilon=20$ ) for fixed $P=40$. But in other cases (both small and large $\varepsilon$ ), there is a tendency of bcc proportion decreasing. It may well explain why the metastable phase of bcc cannot be observed under most of conditions. If we want to observe the liquid-solid phase transition through the metastable bcc phase, the proper choice of the state point will be critical. We will discuss this issue more specifically in Sec. III C.

\section{Transition through metastable bcc}

Now let us concentrate on the case of $\varepsilon=20$ and $P=40$ [see Figs. 5(b) and 6(b)] where the metastable bcc structures are dominant during the process of crystal nucleation. Among the 100 independent samples with different initial configurations, we take a typical one to analyze the dynamic process of the crystallization.

Let us first look at how the number of various particles (including the relatively ordered precursor and crystal particles) evolves during the crystallization, as is displayed in Fig. 6(b). At the early stage of crystallization $(t<5000 \delta t)$, the system runs into a metastable liquid state as there are very few crystal particles but more and more precursors [see the right panel of Fig. 6(b)]. Afterwards, the crystal nucleation starts and the number of solid particles increases apparently until almost all of the particles are crystallized [see the left panel of Fig. 6(b)]. Consistent with Figs. 3(b) and 5(b), both precursors and crystals have predominantly bec-ordered structures.

In order to investigate the crystallization process more clearly, some snapshots which correspond to Fig. 6(b) have been plotted by using the software Visual Molecular Dynamics (VMD) $)^{48}$ (see Fig. 7). Obviously, the nucleation takes place inside the relatively ordered precursor particles, and the precursors are always wetting on the crystals during the whole crystallization. With the growth of nucleus, a bcc crystal is always dominant. Eventually the system can be crystallized into a structure with most bcc particles [see Fig. 7(d)]. For a further confirmation of the crystal structure in the final stage, we calculate the radial distribution function (RDF) which is averaged over the configurations after equilibrium. Comparing with the RDF curve of perfect bcc, we can see that the structure emerged corresponds well with bcc (Fig. 8). This also implies that our crystal identification using the $W_{6}-W_{4}$ plane is suitable.

With fixed $\varepsilon=20$, a set of simulations are performed by varying the pressure. We find that the fractional composition of bcc in the nuclei becomes less and less when increasing the pressure. This can be explained by the Alexander-McTague mechanism as it is often significant for the weakly first-order transition. ${ }^{28} \mathrm{~A}$ high enough pressure ( $\varepsilon$ is fixed) means that

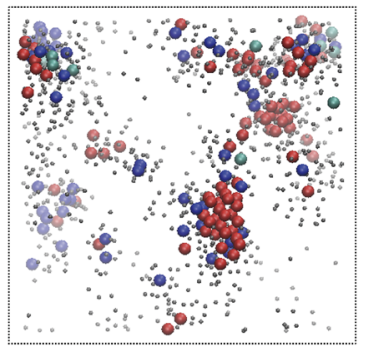

(a)

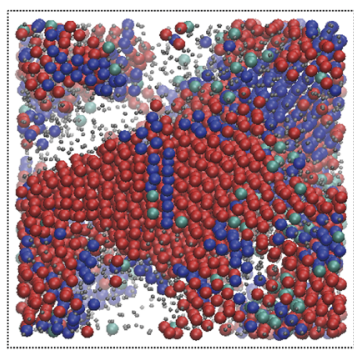

(c)

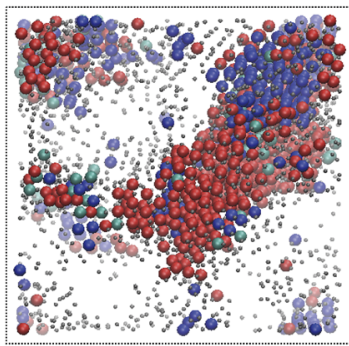

(b)

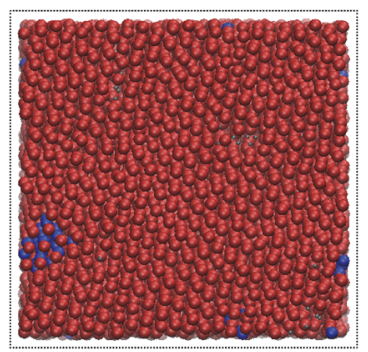

(d)
FIG. 7. Real-space snapshots (plotted via the VMD software ${ }^{48}$ ) of a typical crystal nucleation at the state point of $\varepsilon=20$ and $P=40$. (a) $t=6 \times 10^{3} \delta t$; (b) $t=1.0 \times 10^{4} \delta t$; (c) $t=1.5 \times 10^{4} \delta t$; (d) $t=10^{5} \delta t$. Small spheres represent precursors (liquid particles with $Q_{6} \geq 0.3$ ) while the other liquid particles with $Q_{6}<0.3$ are not displayed for a clearer view. Red, blue, and green big spheres represent bcc, fcc, and hcp particles, respectively.

the state point is so far from the liquid-solid line (see the phase diagram shown in Fig. 1) as to make the AlexanderMcTague mechanism trivial. From the picture of precursor mediated crystallization, the bcc crystal nucleated from the predominantly bcc-ordered precursor is in principle easier due to the symmetry matching because it reduces the interfacial energy. However, for a strong liquid-solid transition (at the state point far from the liquid-solid line where interfacial energy is low enough to be overcome easily), many bcc-ordered precursor particles can directly nucleate into fcc crystals.

For a further study, many more simulations have been performed at other state points with various $\varepsilon, P$, and $\kappa$. If $\varepsilon$ is too small (e.g., $\varepsilon<8$ ), the predominant metastable bcc is not

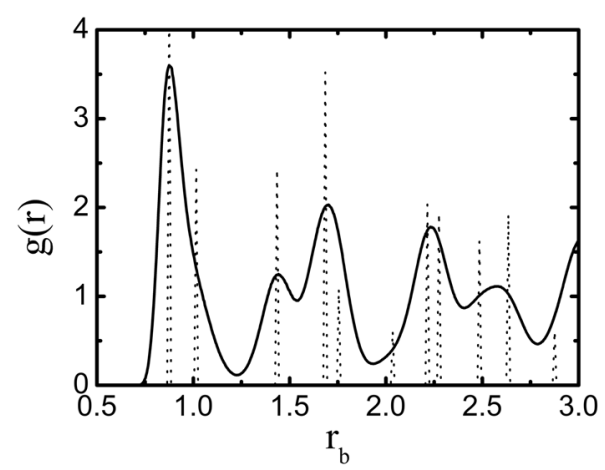

FIG. 8. Averaged RDF curve (solid line) of a typical crystallized system corresponding to Fig. 6(b) ( $t \geq 70000 \delta t$ ), in accord with the RDF of perfect bcc (dotted line). Notice that the pair distance in the $x$ axis is divided by the relevant length scale $L_{0}=\sqrt[3]{\frac{6}{\pi \rho}}$ ( $\rho$ is the number density of systems). 
found at all. Actually the condition of very small $\varepsilon$ indicates that the particles have weak interactions and the characteristic of colloidal system is close to that of hard spheres, so it is no wonder to obtain few bcc crystals during crystallization. When $\varepsilon$ is large enough, the bcc composition decreases with the increasing of pressure, which is similar to the case of $\varepsilon=20$ (related to the weakly first-order transition) and exactly suggests that the dominant bcc crystals are formed only near the bcc-fcc phase boundary (see Fig. 1). The effect of $\kappa$ on the formation of the metastable bcc crystal is similar to that of $\varepsilon$; i.e., only small enough $\kappa$ makes it possible that predominant metastable bcc appears.

Combining all the above discussions together, we can conclude that the formation of dominant metastable bcc needs these necessary conditions: low enough supersaturation or pressure related to the weakly first-order transition, large enough $\varepsilon$, and small enough $\kappa$. In this situation, the state point is always close to the bcc-fcc phase boundary. For the crystallization in most regions of the liquid-solid phase diagram, bcc-ordered precursors have transited to fcc structures before their sizes become macroscopically detectable. It accounts for that the metastable bcc, as pointed out in our previous experiments, ${ }^{14,15}$ can only be seen macroscopically in a narrow window of the colloidal phase diagram. Finally we would like to mention that such a relation between the presence of a fcc-bcc boundary and the crystallization process also happens in other systems, e.g., a similar competition in rare gas systems, ${ }^{49}$ Gaussian core-model system, ${ }^{50}$ and Hertzian spheres. ${ }^{16}$

\section{CONCLUSION}

We have performed the brute-force MD simulations to study the crystal nucleation in charged colloids at different state points where the stable phase is definitely fcc. According to the results, we may conclude that bcc always exists but only dominates in a narrow region. In most of fcc phase regions, the amount of bcc crystal is too small to be detected macroscopically, which is consistent with our experimental finding that there is a rather narrow window of observing the available metastable bcc phase. ${ }^{14,15}$ This also explains why dominant bcc has not been observed in some experiments of charged colloids, e.g., the confocal microscope experiments by Tan et al. ${ }^{12}$ Moreover, we should note that the model of repulsive Yukawa potential differentiates the real colloid-colloid interaction. For instance, the screening length is not constant but dependent on the state point of the system. So the parameters of charged colloids in experiments cannot be tuned as freely as the model used in our simulations, which make the macroscopic detection of the metastable bcc phase even more difficult. In sum, our results suggest a reasonable region where the metastable bcc phase should be detected in macroscopic experiments.

In addition, the formations of preordered precursors and subsequent crystals in charged colloids are investigated. Before a liquid particle transforms into crystal or solid, it is always relatively ordered, i.e., it first becomes a precursor particle with fairly high $\mathrm{Q}_{6}$. Thus the precursors are considered to act as the seeds of crystal nucleation, which is similar to previous findings in many other systems. The structures of precursors, consistent with Ostwald's step rule ${ }^{27}$ and the Alexander-McTague mechanism, ${ }^{28}$ have predominantly bcc-type ordering at all the state points considered. On the other hand, the observations of crystal nucleation suggest that the crystal structures are not determined by the precursors because the composition of crystal formed eventually seems to be dependent on the state point we choose. Such a result tells us that the crystal nucleation mechanism in charged colloids is rather complex and it has more subtle details than expected, which would be interesting for future study.

\section{ACKNOWLEDGMENTS}

This work is supported by Grant Nos. 11572322, 11172302 , and 11672295 from the National Natural Science Foundation of China.

${ }^{1}$ M. E. Davis, Nature 417, 813-821 (2002).

${ }^{2}$ S. Park and R. S. Ruoff, Nat. Nanotechnol. 4, 217-224 (2009).

${ }^{3}$ A. B. Kayitmazer, D. Seeman, B. B. Minsky, P. L. Dubin, and Y. S. Xu, Soft Matter 9, 2553-2583 (2013).

${ }^{4}$ P. G. Vekilov, Prog. Cryst. Growth Charact. Mater. 62, 136-154 (2016).

${ }^{5}$ D. Erdemir, A. Y. Lee, and A. S. Myerson, Acc. Chem. Res. 42, 621-629 (2009).

${ }^{6}$ D. Gebauer, M. Kellermeier, J. D. Gale, L. Bergstrom, and H. Colfen, Chem. Soc. Rev. 43, 2348-2371 (2014).

${ }^{7}$ J. R. Savage and A. D. Dinsmore, Phys. Rev. Lett. 102, 198302 (2009).

8 J. F. Lutsko and G. Nicolis, Phys. Rev. Lett. 96, 046102 (2006).

${ }^{9}$ T. H. Zhang and X. Y. Liu, J. Am. Chem. Soc. 129, 13520-13526 (2007).

${ }^{10}$ T. Schilling, H. J. Schöpe, M. Oettel, G. Opletal, and I. Snook, Phys. Rev. Lett. 105, 025701 (2010).

${ }^{11}$ G. I. Tóth, T. Pusztai, G. Tegze, G. Tóth, and L. Gránásy, Phys. Rev. Lett. 107, 175702 (2011).

${ }^{12}$ P. Tan, N. Xu, and L. Xu, Nat. Phys. 10, 73-79 (2014).

${ }^{13}$ Y. W. Li and Z. Y. Sun, Soft Matter 12, 2009-2016 (2016).

${ }^{14}$ S. H. Xu, H. W. Zhou, Z. W. Sun, and J. C. Xie, Phys. Rev. E 82, 010401 (2010).

${ }^{15}$ H. W. Zhou, Y. M. Qin, S. H. Xu, and Z. W. Sun, Langmuir 31, 7204-7209 (2015).

${ }^{16}$ W. Z. Ouyang, C. L. Fu, Z. W. Sun, and S. H. Xu, Phys. Rev. E 94, 042805 (2016).

${ }^{17}$ W. Z. Ouyang, Z. W. Sun, J. Zhong, H. W. Zhou, and S. H. Xu, Sci. China Chem. 59, 316-323 (2016).

${ }^{18}$ T. Kawasaki and H. Tanaka, Proc. Natl. Acad. Sci. U. S. A. 107, 14036-14041 (2010).

${ }^{19}$ H. Tanaka, J. Phys.: Condens. Matter 23, 284115 (2011).

${ }^{20}$ H. Tanaka, Eur. Phys. J. E 35, 113 (2012).

${ }^{21}$ P. R. ten Wolde, M. J. Ruiz-Montero, and D. Frenkel, Phys. Rev. Lett. 75, 2714-2717 (1995).

${ }^{22}$ P. R. tenWolde, M. J. Ruiz-Montero, and D. Frenkel, J. Chem. Phys. 104, 9932-9947 (1996).

${ }^{23}$ C. Notthoff, B. Feuerbacher, H. Franz, D. M. Herlach, and D. HollandMoritz, Phys. Rev. Lett. 86, 1038-1041 (2001).

${ }^{24}$ D. N. Petsev, X. X. Wu, O. Galkin, and P. G. Vekilov, J. Phys. Chem. B 107, 3921-3926 (2003).

${ }^{25}$ J. Bang and T. P. Lodge, Phys. Rev. Lett. 93, 245701 (2004).

${ }^{26}$ H. J. Schöpe, G. Bryant, and W. van Megen, Phys. Rev. Lett. 96, 175701 (2006).

${ }^{27}$ W. Ostwald, Z. Phys. Chem. 22, 289-330 (1897).

${ }^{28}$ S. Alexander and J. McTague, Phys. Rev. Lett. 41, 702-705 (1978).

${ }^{29}$ J. Russo and H. Tanaka, J. Chem. Phys. 145, 211801 (2016).

${ }^{30}$ J. Russo and H. Tanaka, Soft Matter 8, 4206-4215 (2012).

${ }^{31}$ J. Russo and H. Tanaka, Sci. Rep. 2, 505 (2012).

${ }^{32}$ U. Gasser, E. R. Weeks, A. Schofield, P. N. Pusey, and D. A. Weitz, Science 292, 258-262 (2001).

${ }^{33}$ D. Frenkel, Science 296, 65-66 (2002).

${ }^{34}$ W. Poon, Science 304, 830-831 (2004). 
${ }^{35}$ F. El Azhar, M. Baus, J. P. Ryckaert, and E. J. Meijer, J. Chem. Phys. 112, 5121-5126 (2000).

${ }^{36}$ S. Auer and D. Frenkel, J. Phys.: Condens. Matter 14, 7667-7680 (2002).

${ }^{37}$ K. Kratzer and A. Arnold, Soft Matter 11, 2174-2182 (2015).

${ }^{38}$ C. Desgranges and J. Delhommelle, J. Am. Chem. Soc. 128, 15104-15105 (2006).

${ }^{39}$ L. Y. Gu, S. H. Xu, Z. W. Sun, and J. T. Wang, J. Colloid Interface Sci. 350, 409-416 (2010).

${ }^{40}$ E. J. W. Verwey and J. T. G. Overbeek, Theory of the Stability of Lyophobic Colloids (Elsevier, New York, 1948).

${ }^{41}$ W. Mickel, S. C. Kapfer, G. E. Schröder-Turk, and K. Mecke, J. Chem. Phys. 138, 044501 (2013).

${ }^{42}$ S. Arai and H. Tanaka, Nat. Phys. 13, 503-509 (2017).

${ }^{43}$ W. Lechner and C. Dellago, J. Chem. Phys. 129, 114707 (2008).
${ }^{44}$ G. C. Sosso, J. Chen, S. J. Cox, M. Fitzner, P. Pedevilla, A. Zen, and A. Michaelides, Chem. Rev. 116, 7078-7116 (2016).

${ }^{45}$ H. J. C. Berendsen, J. P. M. Postma, W. F. Vangunsteren, A. Dinola, and J. R. Haak, J. Chem. Phys. 81, 3684-3690 (1984).

${ }^{46}$ D. Frenkel and B. Smit, Understanding Molecular Simulation: From Algorithms to Applications, 2nd ed. (Academic Press, San Diego, 2001).

${ }^{47}$ D. C. Rapaport, The Art of Molecular Dynamics Simulation, 2nd ed. (Cambridge University Press, Cambridge, 2004).

${ }^{48}$ W. Humphrey, A. Dalke, and K. Schulten, J. Mol. Graphics Modell. 14, 33-38 (1996).

${ }^{49}$ C. Desgranges and J. Delhommelle, Phys. Rev. Lett. 98, 235502 (2007).

${ }^{50}$ J. P. Mithen, A. J. Callison, and R. P. Sear, J. Chem. Phys. 142, 224505 (2015). 